\title{
Therapie der erektilen Dysfunktion
}

\section{Von älteren Herrn und neuen Verfahren}

\author{
Nach dem Prototyp eines erektionsfördernden Arzneimittels gefragt, werden die meisten \\ Ärzte an Sildenafil denken. Seit bald 20 Jahren stärkt es Männern in der Liebe den Rücken, \\ obwohl es seinen eigentlichen Effekt deutlich weiter ventral entfaltet.
}

\begin{abstract}
_ Viele Männer, die Sildenafil gegen erektile Dysfunktion (ED) einnehmen, sind bereits von den guten in die besten Jahre gewechselt. Ob sich die Wirkung des Phosphodiesterase-5-Hemmers in den verschiedenen Altersgruppen unterscheidet, haben Sexualmediziner um Irwin Goldstein vom Alvarado Hospital in San Diego untersucht. Ihre Ergebnisse haben die Forscher auf dem 21. Herbstmeeting der Sexual Medicine Society of North America vorgestellt und nun im Journal of Sexual Medicine publiziert [1]
\end{abstract}

\section{Metaanalyse von 48 Studien}

Goldstein und Kollegen bedienten sich für ihre Studie des umfangreichen Datenmaterials, das in diversen Studien mit Sildenafil gesammelt worden ist. Dazu poolten sie die Angaben zu mehr als 11.000 Männern mit ED, die an 48 randomisierten und placebokontrollierten Doppelblindstudien teilgenommen hatten.

\section{Braucht er Hilfe?}

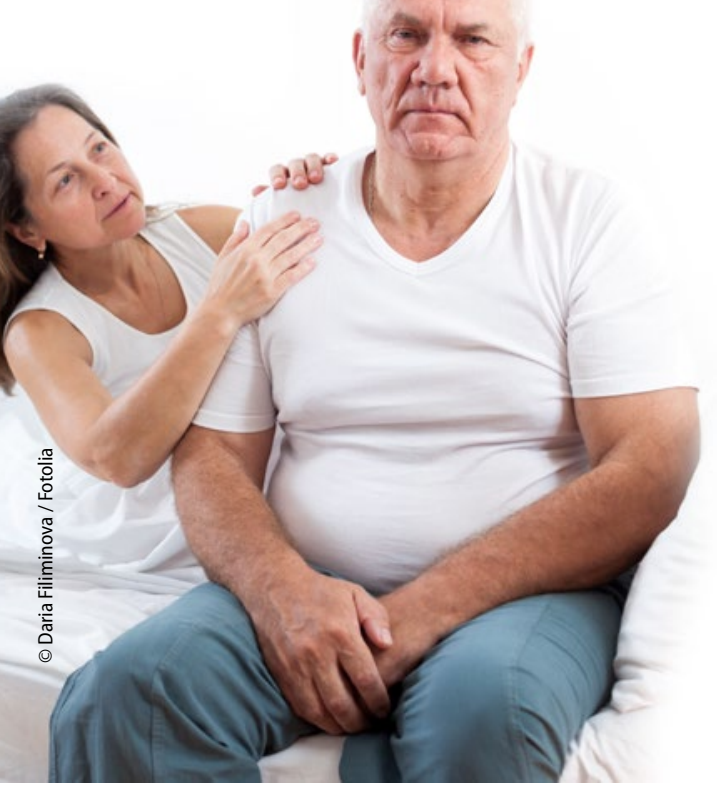

Die meisten der analysierten Studien hatten zwölf Wochen gedauert. Die Startdosis hatte $50 \mathrm{mg}$ Sildenafil betragen, einzunehmen eine Stunde vor dem Zeitpunkt der benötigten Wirkung. Abhängig von Effekt und Nebeneffekten wurde die Dosis anschließend auf 100 mg erhöht oder auf 25 mg gesenkt.

\section{Verbesserte Erektion im Placebovergleich}

Als Maßstab der Wirkung diente zum einen der International Index of Erectile Function. Die erektile Funktion - speziell die Fähigkeit zur Penetration und zum Aufrechterhalten der Erektion zeigte sich unter Sildenafil im Vergleich zu Placebo in allen drei untersuchten Altersgruppen $(<65 ; 65-74 ; \geq 75$ Jahre $)$ verbessert.

Auf die Frage „Hat die Behandlung ihre Erektionen verbessert?“ antworteten nach zwölf Wochen in der Gruppe unter 65 Jahren $80 \%$, von den 64 - bis 74-Jährigen $69 \%$ und von den älteren Männern 59\% mit „Ja“. Das waren in allen Gruppen höhere Anteile als unter Placebo, wenngleich mit zunehmendem Alter eine Tendenz zu abnehmender $\mathrm{Zu}$ friedenheit zu verzeichnen war.

„Sildenafil ist eine wirksame und gut verträgliche Therapie für Männer mit erektiler Dysfunktion“, folgern Goldstein und Mitarbeiter aus ihren Ergebnissen. Das Alter spiele dabei keine große Rolle, auch die meisten Männer ab 75 müssten solcherart unterstützt nicht auf Sex verzichten.

\section{Alternative Zubereitungsformen}

Pflaster ...

Derweil wird andernorts an alternativen Zubereitungsformen von Sildenafil gearbeitet. Die Gründe dafür sind eine relativ geringe Bioverfügbarkeit (ca. $40 \%)$ und ein relativ hoher First-PassEffekt (70\% der Dosis) nach oraler Einnahme. Außerdem dauert es rund eine Stunde, bis das Mittel wirkt, nach einer fettreichen Mahlzeit eventuell noch länger. Eine arabische Pharmazeutenriege arbeitet daher an einem transdermalen Freisetzungssystem für Sildenafil nach Art eines Pflasters [2]. Das Verfahren soll vor allem die Bioverfügbarkeit erhöhen.

\section{... oder Sublingualspray}

Taiwanische Pharmakologen forschen derweil an einer Galenik, die einen schnelleren Wirkbeginn ermöglichen soll [3]. Sie hat die Form eines sublingualen Sprays. Sildenafilcitrat wird in Ölsäure oder Propylenglykol emulgiert und unter die Zunge gesprüht oder das granulierte Spray auf ein Silikatadsorbens aufgebracht und als Sublingualtablette eingenommen. Die Wirkung setzt hier offenbar binnen Minuten ein und hält rund eineinhalb Stunden an.

Beide Prinzipien - Pflaster und sublinguale Applikation - sind in vivo erfolgreich getestet worden. Das Pflaster kam Ratten, das sublinguale Spray Kaninchen zugute. Ob die neuen Galeniken eines Tages auch Männern eine Hilfe sein werden, muss noch offen bleiben. Dass aber selbst Karnickel von Sildenafil profitieren, ist immerhin eine originelle Erkenntnis.

Dr. Robert Bublak

\footnotetext{
- 1. Goldstein l et al. Efficacy and Safety of Sildenafil by Age in Men With Erectile Dysfunction. J Sex Med 2016;13(5):852-9 2. Badr-Eldin SM, Ahmed OAA. Optimized nano-transferosomal films for enhanced sildenafil citrate transdermal delivery: ex vivo and in vivo evaluation. Drug Des Devel Ther 2016; 10:1323-33

3. Sheu MT et al. Rapid-Onset Sildenafil Sublingual Drug Delivery Systems: In Vitro Evaluation and In Vivo Pharmakokinetic Studies in Rabbits. J Pharm Sci 2016, online 14. März; doi: 10.1016/j.xphs.2016.01.015
} 